\title{
Effect of propranolol with and without rosuvastatin on migraine attacks: a triple blind randomized clinical trial
}

\author{
Mehrdokht Mazdeh¹, Rihane Mahmudian², Seyed Y Vafaei ${ }^{3}$, Mohammad Taheri*,4 (iD \& \\ Soudeh Ghafouri-Fard**,5 (D) \\ ${ }^{1}$ Neurophysiology Research Center, Hamadan University of Medical Sciences, Hamadan, Iran \\ ${ }^{2}$ Department of Neurology, Hamadan University of Medical Sciences, Hamadan, Iran \\ ${ }^{3}$ Department of Pharmaceutics \& Pharmaceutical Biotechnology, School of Pharmacy, Hamadan University of Medical Sciences, \\ Hamadan, Iran \\ ${ }^{4}$ Urogenital Stem Cell Research Center, Shahid Beheshti University of Medical Sciences, Tehran, Iran \\ ${ }^{5}$ Department of Medical Genetics, Shahid Beheshti University of Medical Sciences, Tehran, Iran \\ *Author for correspondence: Tel. \& Fax: +00982123972572; mohammad_823@yahoo.com \\ **Author for correspondence: s.ghafourifard@sbmu.ac.ir
}

\begin{abstract}
Aim: To investigate the effect of rosuvastatin in combination with propranolol in reducing migraine attacks. Patients \& methods: In a clinical trial study with census method, 120 patients with migraine headache were included. Patients were randomly assigned (using block randomization) to either propranolol (10 mg twice a day) with rosuvastatin (10 mg daily; intervention group [ $\mathrm{n}=60]$ ) or propranolol (10 mg twice a day) with placebo (control $[n=60]$ ). Results: The numbers of attacks were significantly decreased in the intervention group at the end of second, third and fourth weeks from the beginning of the intervention ( $p<0.05)$. Conclusion: In patients with migraine, concomitant administration of propranolol (10 $\mathrm{mg}$ twice a day) and rosuvastatin (10 $\mathrm{mg}$ daily) is effective in reducing migraine attacks.
\end{abstract}

First draft submitted: 14 December 2019; Accepted for publication: 11 February 2020; Published online: 18 March 2020

Keywords: migraine $\bullet$ propranolol $\bullet$ rosuvastatin

Migraine is one of the most frequent disorders and an important source of disability. This neurological condition has imposed an extensive clinical and financial burden on both the patient and society [1]. Migraine attacks are characterized by a wide range of sensory processing abnormalities leading to headaches, photophobia, phonophobia, osmophobia and nausea [2]. While previous investigations have detected a vascular origin for migraine [3], more recent studies have highlighted the role of cortical spreading depression, extravasation of neurogenic plasma protein and dysfunction of the brainstem in the pathogenesis of migraine or the associated symptoms [2]. Yet, the most accepted theory about migraine is the activation of the trigeminovascular system [4]. Several different medications have been suggested to alter the migraine attacks underlying pathogenic events to prevent or treat these attacks [5]. Beta-blockers have been frequently used as the first choice for the prevention of migraines [6]. While the exact mechanism of function of $\beta$-blockers in migraine prophylaxis is not clear, their influence on the catecholaminergic system and serotonin receptors in the CNS could be a possible function [7]. Propranolol is one of the most frequently prescribed $\beta$-blockers for migraine attack prevention [8,9]. A recent meta-analysis of randomized controlled trials has shown the efficacy of propranolol in controlling the episodic migraine headaches [10]. Statins have been also recently tested for the prevention of migraine attacks. This group of cholesterol-lowering drugs exhibits some cholesterol-independent functions such as amending endothelial dysfunction [11], decreasing inflammatory responses in vessels [12], reduction of platelet aggregation [13] and improvement of the function of the autonym nervous system [14]. Administration of simvastatin in conjunction with vitamin D has been proven to effectively prevent migraine attacks in adult patients [15]. Statins have been suggested as a useful medication in migraine management since they could decrease the elevated cardiovascular risk in migraineurs, hence reducing the migraine attacks [16]. Moreover, a recent study has suggested atorvastatin as an alternative for sodium valproate in the 
prevention of migraine attacks with similar efficacy and lower complications [17]. Besides, results of animal studies have introduced atorvastatin as a promising candidate for migraine treatment or prevention via decreasing NF- $\kappa \mathrm{B}$ signaling in trigeminal nucleus caudalis [18]. Additionally, statins can suppress potassium channels that according to recent studies are linked to migraine pathophysiology [19].

The aim of the present study is to assess the efficacy of combination therapy including rosuvastatin and propranolol to prevent migraine attacks through a randomized, triple-blind, placebo-controlled study design.

\section{Patients \& methods}

The study was conducted in Imam Khomeini Clinic and Sina hospital, Hamadan, Iran, from January 2018 to September 2019. Vitamin D levels were assessed in all the studied participants at the baseline using the Euroimmun ELISA (the enzyme-linked immunosorbent assay) kit (Germany).

\section{Eligibility criteria}

The convenience nonprobability method was used for sampling. Sampling was continued for 8 months. During this time period, 120 patients who met the criteria entered the study. The inclusion criteria were as follow: age $\geq 18$ years, more than 3 years history of migraine, presence of migraine attacks $\geq 4$ days/month, total cholesterol levels between 150 and $190 \mathrm{mg} / \mathrm{dl}$. The diagnosis of migraine was accomplished according to the International Headache Society for headache classification (ICHD-III) criteria [20]. Exclusion criteria were pregnancy, breastfeeding, hepatic or renal failure, memory defects, sensitivity to statins and presence of atherosclerotic disorders.

\section{Intervention}

Patients were randomly assigned to intervention and control groups. As we wanted to increase compliance of patients to the prescribed drugs and avoid self-prescribed medications, we did not include the third arm with placebo and placebo 'nothing arm'. A block randomization method was utilized to randomize the patients into two groups with equal sizes. Random allocation sequence, enrollment of participants and assignment of participants to groups were performed by trained staff. The patients, the physicians who assessed the patients, and the staff who analyzed the data were not aware of patients' status until the end of the study as they were separated from those involved in the patients' allocation process. The study was designed as a triple-blind study. The intervention group $(n=60)$ received propranolol (10 mg film-coated tablets, twice a day, dosage form: $10 \mathrm{mg}$ tablet $)$ and rosuvastatin (10 mg film-coated tablets, daily, dosage form: $10 \mathrm{mg}$ tablet); while the control group $(n=60)$ were treated with propranolol (10 mg film-coated tablets, twice a day, dosage form: $10 \mathrm{mg}$ tablet) and placebo. Special diets and aerobic exercise were recommended for both groups and the number of migraine attacks was monitored for 4 weeks. No rescue medications were used for acute migraine attacks. No concomitant medication was allowed during the study period. Questionnaires (Supplementary Data) were designed by the research team and filled by the trained staff via telephone contacts with patients at the end of each week during the study period (4 weeks). The numbers of migraine attacks were calculated by asking from patients. All patients had pain-free intervals. The frequency of attacks was assessed accordingly. Compliance with medications was checked by the staff during telephone contacts. The patients were on a migraine diary. Yet, patients were not on prophylactic migraine medication at baseline. Patients were contacted at the end of each week to assess their possible complications, number of migraine attacks and compliance to medications. The study protocol was approved by ethical committee of Hamadan University of Medical Sciences. Written consent forms were signed by study participants and permission to use human data was obtained. The clinical trial number was IRCT20120215009014N267. Figure 1 shows the study flowchart.

\section{Primary outcome}

Numbers of migraine attacks per month were obtained through an interview with the patients.

\section{Secondary outcomes}

Secondary outcome was assessment of the side effects such as hepatic failure, gastrointestinal complications, memory defects, myopathy and hypersensitivity to statins. Safety was described by occurrence of clinically observable side effects. 


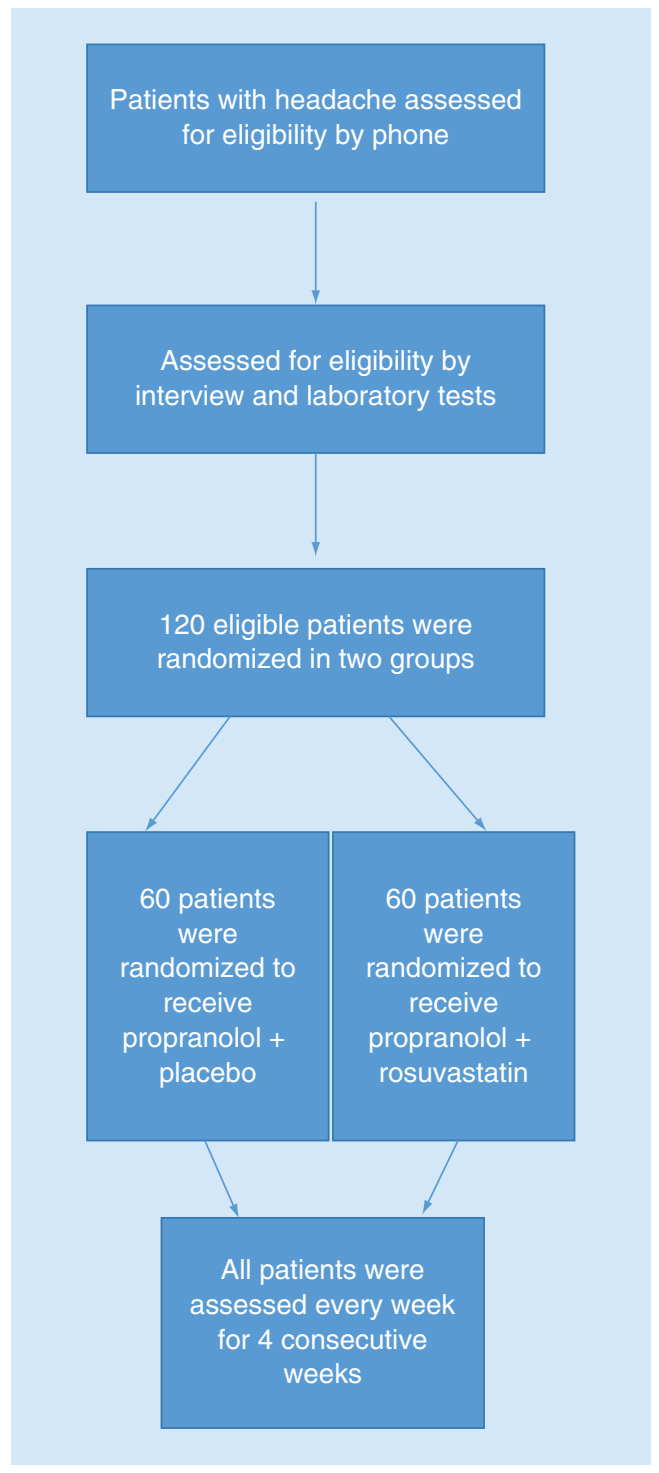

Figure 1. Study flowchart. Assessment of patients' eligibility was a stepwise process including assessments through phone calls, interview and laboratory tests. Then, the 120 eligible persons were randomly assigned to intervention and control groups, respectively.

\section{Statistical methods}

Data analysis was conducted using SPSS v. 21 (IBM Corp., IL, USA). All the analyses were estimated at 95\% CIs. p-values below 0.05 were regarded as significant. Categorical variables were described as ratios or percentages and shown in tables and diagrams. The normality of the frequency of migraine attacks was assessed using the Kolmogorov-Smirnov test. Mean values of the frequency of migraine attacks were compared using the MannWhitney test. Mean values of migraine attacks per month based on the vitamin D levels were also compared using the Kruskal-Wallis test and Tukey post hoc test.

\section{Results}

\section{Baseline characteristics of study participants}

Each of the intervention and control groups included 60 individuals with similar sex ratios (female/male: 56 [93.3\%]/4 [6.7\%]). The study groups were also matched in terms of age (mean age \pm standard deviation [SD] values: $36.27 \pm 10.58$ and $33.72 \pm 10.16$ in the control and intervention groups, respectively; $p=0.185)$. Besides, the participants showed no difference in their serum levels of vitamin D ( $\mathrm{p}=0.125)$. Table 1 shows baseline characteristics of patients in each group. There was no dropout during the study period. 


\begin{tabular}{|c|c|c|}
\hline Parameters & Control group & Intervention group \\
\hline Age $($ mean $\pm S D)$ & $36.27 \pm 10.58$ & $33.72 \pm 10.16$ \\
\hline Sex ratio (female/male) & $56(93.3 \%) / 4(6.7 \%)$ & $56(93.3 \%) / 4(6.7 \%)$ \\
\hline \multicolumn{3}{|l|}{ Vitamin D levels: } \\
\hline - Deficient & $23(38.3 \%)$ & $34(56.7 \%)$ \\
\hline - Insufficient & $10(16.7 \%)$ & $6(10.0 \%)$ \\
\hline - Sufficient & $27(45.0 \%)$ & $20(33.3 \%)$ \\
\hline - Sum & $60(100 \%)$ & $60(100 \%)$ \\
\hline
\end{tabular}

\begin{tabular}{|c|c|c|c|}
\hline Time point & Control group & Intervention group & $p$-value ${ }^{\dagger}$ \\
\hline First week & $2.56 \pm 2.69$ & $1.90 \pm 2.06$ & 0.356 \\
\hline Second week & $2.27 \pm 2.35$ & $1.46 \pm 1.96$ & 0.022 \\
\hline Third week & $2.24 \pm 2.26$ & $1.14 \pm 1.69$ & 0.008 \\
\hline Fourth week & $2.53 \pm 2.38$ & $1.00 \pm 1.80$ & $<0.001$ \\
\hline
\end{tabular}

\begin{tabular}{|c|c|c|}
\hline Vitamin D levels & Number of attacks & p-value \\
\hline Deficient & $6.84 \pm 7.24$ & 0.002 \\
\hline Insufficient & $8.33 \pm 7.81$ & \\
\hline Sufficient & $2.35 \pm 4.26$ & \\
\hline
\end{tabular}

Four individuals in the control group (6.7\%) and one in the intervention group (1.7\%) had aura. Based on the results of the Fisher exact test, no difference was detected between the studied groups in terms of this issue $(\mathrm{p}=$ $0.171)$.

At the beginning of the study, the mean $( \pm S D)$ values of migraine attacks per week were $4.38( \pm 2.39)$ and 4.05 $( \pm 2.09)$ in control and intervention groups, respectively. Based on the Mann-Whitney test results, there was a difference in the number of migraine attacks between the studied groups $(\mathrm{p}=0.872)$.

\section{Primary measures}

Mean numbers of migraine attacks per week in the intervention and control groups were 1.90 versus $2.56(\mathrm{p}=$ $0.356), 1.46$ versus $2.27,1.14$ versus $2.24(\mathrm{p}=0.008)$ and 1.00 versus $2.53(\mathrm{p}<0.001)$, at the end of the first to fourth weeks of the study, respectively. No significant difference was recorded in the number of migraine attacks between the two groups at the end of the first week following the intervention. However, the numbers of attacks were significantly declined in the intervention group at the end of the second, third and fourth weeks. Table 2 shows the results of comparisons.

Based on the results of repeated measures tests, numbers of migraine attacks were different between study groups after 4 weeks.

Based on the results obtained from Kruskal-Wallis and Tukey post hoc tests, the number of migraine attacks per month was significantly lower in the patients with sufficient vitamin D levels as compared with those suffering from deficient vitamin $\mathrm{D}$ levels $(\mathrm{p}=0.016)$. However, 'sufficient versus insufficient' and 'insufficient versus deficient' comparisons showed no significant differences ( $\mathrm{p}$-values $=0.051$ and 0.607 , respectively). Rosuvastatin treatment did not significantly alter vitamin $\mathrm{D}$ levels in the intervention group $(\mathrm{p}=0.4)$.

Table 3 shows the number of migraine attacks in the study period in intervention group based on vitamin $\mathrm{D}$ levels. 


\section{Secondary measures}

Routine physical examinations and history taking were carried out to assess the possible side effects. There was no report of statin-associated side effects such as hepatic failure, gastrointestinal complications, memory defects, myopathy or hypersensitivity in patients.

\section{Discussion}

In the present triple-blind randomized clinical trial, the effect of rosuvastatin coadministration with propranolol on decreasing the number of migraine attacks was assessed. In this study, we used low-dose propranolol to prevent side effects such as hypotension. The study period was shorter than studies, which assess efficacy of medications in migraine prophylaxis. However, as there was no similar study in the literature, we aimed at identification of short time effects of rosuvastatin in migraine prophylaxis. In comparison with single propranolol therapy, rosuvastatin and propranolol coadministration significantly declined the number of migraine attacks. Moreover, migraine attacks were significantly more frequent in patients with insufficient vitamin D levels as compared with those having sufficient vitamin D highlighting the impact of adequate vitamin $\mathrm{D}$ on ameliorating migraines.

Previous studies have reported a higher prevalence of cardiovascular disorders among migraineurs. A large, prospective cohort study by Kurth et al. in female migraineurs showed a reliable association between migraine and cardiovascular disease and cardiovascular disease-induced mortalities [21]. Moreover, migraine has been recognized as an inflammatory condition caused by stimulation of the trigeminal neurovascular complex; therefore, statins could be beneficial in this disorder especially for patients with aura [22]. A recent meta-analysis of 16 cohort studies has verified the association between migraine and higher long-term risk of cardiovascular and cerebrovascular events [23]. In this regard, statins can be helpful in the treatment of migraine patients by decreasing the risk of cardiovascular events. In a randomized, double-blind, placebo-controlled trial, Buettner et al. reported the efficacy of simvastatin plus vitamin D3 in the prevention of headaches in adults with episodic migraines [15]. Moreover, a previous cross-sectional study revealed the association of statin with a lower incidence of severe headache or migraines, especially in those with higher serum vitamin D levels [24]. This observation is in line with our results regarding the higher efficacy of rosuvastatin in reducing migraine attacks in patients with sufficient vitamin D levels. According to these studies, patients' exposure to statins might decline migraine-associated cardiovascular events. Therefore, the increase in the cost and chances of statin-related adverse events could be compensated by the reduced risk of cardiovascular events. This note is highlighted by the observed higher frequency of dyslipidemia in migraineurs compared with controls as a recent study reported high cholesterol and LDL levels in 42 and 44\% of migraineurs, respectively; whereas these values were $24 \%$ among the controls [25]. It is worth mentioning that statin are regarded as safe drugs as the risk of statin-induced serious muscle injury, serious hepatotoxicity and statin-induced newly diagnosed diabetes mellitus are negligible. Moreover, the great statin-associated decrease in the risk of atherothrombotic stroke, total stroke and other cardiovascular events is considerably more than possible side effects of these drugs [25]. Meanwhile, safety profile of propranolol in various conditions have been reported to be acceptable [26].

No significant change was observed in vitamin D levels of the intervention group. Some previous studies demonstrated the role of rosuvastatin in increasing the serum vitamin D levels [27], yet others challenged this observation and stated that these results need to be confirmed in well-designed prospective studies in which the effects of confounding factors such as ultraviolet- $B$ irradiance and oral vitamin $D$ supplementations are controlled [20]. Thus, there is no general consensus on this point. However, if effects of rosuvastatin on serum vitamin $\mathrm{D}$ levels are verifies, this drug can be regarded as a medication that ameliorates migraine via different mechanisms.

Our study had some limitations regarding the follow-up period and sample size. In this context, the verification of the obtained results by larger cohorts of patients and for longer periods is highly encouraged. Moreover, we have not reported data of mean migraine days and vitamin D levels at follow-up, which is recommended to be assessed in future studies.

\section{Conclusion}

Taken together, our results support the beneficial effects of a certain statin namely rosuvastatin in reducing migraine attacks in Iranian patients. As statins can amend endothelial dysfunction through different mechanisms [28], the administration of rosuvastatin might also decrease the elevated risk of vascular diseases among patients suffering from migraines. Based on the higher efficacy of rosuvastatin in reducing migraine attacks in patients with sufficient 
vitamin $\mathrm{D}$ levels, further clinical trials are recommended to test the effects of rosuvastatin plus vitamin $\mathrm{D}$ in migraineurs.

\section{Future perspective}

Based on the extensive clinical and financial burden of migraine, future studies should focus on introduction of novel strategies for prevention of migraine headache. Moreover, safety of the prescribed medications should be assessed through laboratory tests.

\section{Summary points}

- We investigated the effect of rosuvastatin in combination with propranolol in reducing migraine attacks.

- Each of the intervention and control groups included 60 individuals with similar sex ratios.

- Baseline characteristics were matched between study groups at the beginning of the study.

- There was no significant difference in number of migraine attacks between two groups at the end of the first week following intervention.

- The numbers of attacks were significantly decreased in the intervention group at the end of second, third and fourth weeks from the beginning of the intervention.

- Numbers of migraine attacks were significantly higher in patients with insufficient vitamin $D$ levels compared with those having sufficient vitamin D.

- In patients with migraine, concomitant administration of propranolol (10 mg twice a day) and rosuvastatin (10 mg daily) is effective in reducing migraine attacks.

- Based on the higher frequency of cardiovascular event among migraineurs, administration of statins might be beneficial in these patients.

Financial \& competing interests disclosure

The current study was supported from Hamadan University of Medical Sciences. The authors have no other relevant affiliations or financial involvement with any organization or entity with a financial interest in or financial conflict with the subject matter or materials discussed in the manuscript apart from those disclosed.

No writing assistance was utilized in the production of this manuscript.

\section{Ethical conduct of research}

The study protocol was approved by ethical committee of Hamadan University of Medical Sciences. Written consent forms were signed by study participants and permission to use human data was obtained. The authors state that they have obtained appropriate institutional review board approval or have followed the principles outlined in the Declaration of Helsinki for all human or animal experimental investigations. In addition, for investigations involving human subjects, informed consent has been obtained from the participants involved.

Data sharing statement

The authors certify that this manuscript reports original clinical trial data, IRCT20120215009014N267. Individual participant data that underlie the results reported in the article, after deidentification (text, tables, figures and supplement) are available along with the study protocol and statistical analysis. The data will be available from the publication date up until 5 years. Proposals to access data will be at the discretion of the corresponding author and to gain access, the data requestor will need to sign a data access agreement form.

Open access

This work is licensed under the Attribution-NonCommercial-NoDerivatives 4.0 Unported License. To view a copy of this license, visit http://creativecommons.org/licenses/by-nc-nd/4.0/

\section{References}

Papers of special note have been highlighted as: $\bullet$ of interest

1. Agosti R. Migraine burden of disease: from the patient's experience to a socio-economic view. Headache 58(Suppl. 1), 17-32 (2018).

2. Sprenger T, Goadsby PJ. Migraine pathogenesis and state of pharmacological treatment options. BMC Med. 7, 71 (2009).

- A comprehensive description of pathogenesis of migraine. 
3. Moskowitz MA, Buzzi MG, Sakas DE, Linnik MD. Pain mechanisms underlying vascular headaches. Progress report 1989. Rev. Neurol. (Paris) 145(3), 181-193 (1989).

4. Noseda R, Burstein R. Migraine pathophysiology: anatomy of the trigeminovascular pathway and associated neurological symptoms, CSD, sensitization and modulation of pain. Pain 154(Suppl.1), S44-S53 (2013).

- A comprehensive description of pathogenesis of migraine.

5. Goadsby PJ. Pathophysiology of migraine. Ann. Indian Acad. Neurol. 15(Suppl. 1), S15-S22 (2012).

- A comprehensive description of pathogenesis of migraine.

6. Pringsheim T, Davenport W, Mackie G et al. Canadian Headache Society guideline for migraine prophylaxis. Can. J. Neurol. Sci. 39 (2 Suppl. 2), S1-S59 (2012).

7. Pompili M, Serafini G, Innamorati M et al. Patient outcome in migraine prophylaxis: the role of psychopharmacological agents. Patient Relat. Outcome Meas. 1, 107-118 (2010).

8. Linde K, Rossnagel K. Propranolol for migraine prophylaxis. Cochrane Database Syst. Rev. (2), CD003225 (2004).

9. al-Qassab HK, Findley LJ. Comparison of propranolol LA $80 \mathrm{mg}$ and propranolol LA $160 \mathrm{mg}$ in migraine prophylaxis: a placebo controlled study. Cephalalgia 13(2), 128-131 (1993).

10. Jackson JL, Kuriyama A, Kuwatsuka Y et al. Beta-blockers for the prevention of headache in adults, a systematic review and meta-analysis. PLoS ONE 14(3), e0212785 (2019).

11. Antoniades C, Bakogiannis C, Leeson P et al. Rapid, direct effects of statin treatment on arterial redox state and nitric oxide bioavailability in human atherosclerosis via tetrahydrobiopterin-mediated endothelial nitric oxide synthase coupling. Circ. 124(3), 335-345 (2011).

12. Forero-Peña DA, Gutierrez FR. Statins as modulators of regulatory T-cell biology. Mediators Inflamm. 167086 (2013).

- A brief description of the role of statins in regulation of immune responses.

13. Liao JK, Laufs U. Pleiotropic effects of statins. Ann. Rev. Pharmacol. Toxicol. 45, 89-118 (2005).

14. Millar PJ, Floras JS. Statins and the autonomic nervous system. Clin. Sci. 126(6), 401-415 (2014).

15. Buettner C, Nir RR, Bertisch SM et al. Simvastatin and vitamin D for migraine prevention: a randomized, controlled trial. Ann. Neurol. 78(6), 970-981 (2015).

16. Liberopoulos EN, Mikhailidis DP. Could statins be useful in the treatment of patients with migraine? Headache 46(4), 672-675 (2006).

\section{- A brief description of the role of statins in control of headache.}

17. Hesami O, Sistanizad M, Asadollahzade E, Johari MS, Beladi-Moghadam N, Mazhabdar-Ghashghai H. Comparing the effects of atorvastatin with sodium valproate (divalproex) on frequency and intensity of frequent migraine headaches: a double-blind randomized controlled study. Clin. Neuropharmacol. 41(3), 94-97 (2018).

18. Yin Z, Fang Y, Ren L et al. Atorvastatin attenuates NF-kappaB activation in trigeminal nucleus caudalis in a rat model of migraine. Neurosci. Lett. 465(1), 61-65 (2009).

19. Al-Karagholi MA, Hansen JM, Guo S, Olesen J, Ashina M. Opening of ATP-sensitive potassium channels causes migraine attacks: a new target for the treatment of migraine. Brain 142(9), 2644-2654 (2019).

20. Levin M. The international classification of headache disorders, (ICHD III) - changes and challenges. Headache 53(8), 1383-1395 (2013).

21. Kurth T, Winter AC, Eliassen AH et al. Migraine and risk of cardiovascular disease in women: prospective cohort study. BMJ 353, i2610 (2016).

22. Kernick D. Statins for all: should patients who have migraine with aura be on a statin? Br. J. Gen. Pract. 65(640), 571-572 (2015).

23. Mahmoud AN, Mentias A, Elgendy AY et al. Migraine and the risk of cardiovascular and cerebrovascular events: a meta-analysis of 16 cohort studies including 1152407 subjects. BMJ Open 8(3), e020498 (2018).

24. Buettner C, Burstein R. Association of statin use and risk for severe headache or migraine by serum vitamin D status: a cross-sectional population-based study. Cephalalgia 35(9), 757-766 (2015).

25. Newman CB, Preiss D, Tobert JA et al. Statin safety and associated adverse events: a scientific statement from the American Heart Association. Arterioscler. Thromb. Vasc. Biol. 39(2), e38-e81 (2019).

26. Srinivasan AV. Propranolol: a 50-year historical perspective. Ann. Indian Acad. Neurol. 22(1), 21-26 (2019).

27. Ertugrul DT, Yavuz B, Cil H et al. STATIN-D study: comparison of the influences of rosuvastatin and fluvastatin treatment on the levels of 25 hydroxyvitamin D. Cardiovasc. Therap. 29(2), 146-152 (2011).

28. Altun I, Oz F, Arkaya SC et al. Effect of statins on endothelial function in patients with acute coronary syndrome: a prospective study using adhesion molecules and flow-mediated dilatation. J. Clin. Med. Res. 6(5), 354-361 (2014). 